\title{
ENTRE EUROPA Y AMERICA LATINA: LA DIPLOMACIA ESPAÑOLA FRENTE AL CONFLICTO DE LAS MALVINAS
}

\author{
Esther Barbé
}

La postura de España frente a la guerra de las Malvinas se caracteriza por su ambigüedad y por ser contraria a los países de su entorno. Esto se debe a las circunstancias internas del momento y a que los temas prioritarios de la agenda-descolonización de Gibraltar, ingreso en las Comunidades Europeas y mantenimiento de relaciones privilegiadas con América Latina-no pueden implementarse conjuntamente. Sin embargo, lo comunitario domina claramente y, así, el proceso de incorporación a las instituciones europeas es determinante. Para comprender lo anterior, primeramente se explica el estilo de toma de decisiones en la España democrática. Luego, se describe su posición ante el conflicto y se analiza el debate político existente. Se comprueba, entonces, la "inoportunidad" de la guerra para los intereses españoles y se extraen lecciones generales sobre sus opciones en materia internacional. Finalmente, se concluye que en la última década España ha burocratizado sus relaciones con América Latina y que en situaciones de crisis de estos países todavía actúa como un disidente potencial.

En los últimos años han abundado los estudios sobre política exterior española y, en concreto, sobre las relaciones entre la España postfranquista y América Latina. Es difícil encontrar en dichos estudios alguna mención a la guerra de las Malvinas. Se diría que este conflicto armado apenas provocó reacción por parte de España. Este artículo intenta analizar dicha reacción (incluyendo Gobierno, fuerzas políticas y sociales, prensa y opinión pública), a la luz de una pregunta pendiente: ¿en qué medida se ve afectada la diplomacia española, durante la guerra de las Malvinas, por el proceso de incorporación del país a las instituciones europeas? ${ }^{1}$ En otras palabras, ¿́cómo se

\footnotetext{
${ }^{1}$ La versión inglesa de este artículo forma parte de un libro colectivo Ch. Hill y S. Stavridis (eds.), Domestic Sources of Foreign Policy: West European Reactions to the Falklands Conflict, (Oxford: Berg Publishers, 1994), en el que se aborda la posición comparada de los países comunitarios más destacados durante la guerra (los grandes países o los países divergentes con la política colectiva de los Diez), más España y la Comunidad Europea, en su conjunto.
} 
hubiera comportado España en el marco de la Cooperación Política Europea? Por una parte, no hay que olvidar que los.Diez (miembros de la Comunidad) llevaron a cabo durante la guerra de las Malvinas una de sus políticas más "duras" hasta aquel momento, con adopción de sanciones económicas (Edwards, 1984). Por otra parte, la incorporación de España al mecanismo diplomático de la Comunidad (Cooperación Política Europea) se realizó con gran fervor europeísta, lo que convirtió a España en un activista en favor de la "diplomacia europea" (Barbé, 1990). De ahí que dejemos planteada la pregunta, como "hilo conductor": ¿qué posición hubiera adoptado España durante la guerra de las Malvinas, caso de haber sido ya miembro de la Comunidad?

De entrada, hay que apuntar que la posición española durante la guerra constituye un caso particular, en comparación con los países de su entorno geopolítico (los países comunitarios más Portugal). Hay que empezar, sin embargo, señalando que España por sí misma constituye en 1982 un caso particular en relación a los países de su entorno. La combinación de tres elementos otorga a España ese carácter particular en 1982:

1.- La prioridad absoluta de la política interna sobre la política exterior. La España de 1982 vive los últimos momentos de la transición política de la dictadura franquista a la democracia. De hecho, las elecciones del 28 de octubre de 1982 que gana, con mayoría absoluta, el Partido Socialista Obrero Español (PSOE) significan la consolidación del sistema democrático instaurado con la Constitución de 1978 (Morodo, 1984). Durante la guerra de las Malvinas la clase política española está centrada en las divergencias que dividen al partido gobernante (Unión de Centro Democrático, UCD) y que paralizan la actuación del ejecutivo, se halla a la espera de las sentencias contra los golpistas del 23 de febrero ${ }^{2} \mathrm{y}$, obviamente, está preparando las elecciones del mes de octubre.

2.- La agenda diplomática del Gobierno español se encuentra en el momento en que comienza la guerra de las Malvinas ocupada por el proceso de ingreso del país en la Alianza Atlántica (ratificación por parte de los quince miembros de la OTAN de la adhesión española), que finalizará el 30 de mayo. Dicho ingreso es una más de las consecuencias internacionales de la transición política en España. En efecto, la transición comporta la redefinición del papel de España en

\footnotetext{
${ }^{2}$ Se refiere a los militares participantes en el abortado golpe de Estado del 23 de febrero de 1981.
} 
las relaciones internacionales. Lo que en abril de 1982 se traduce en que el Gobierno de Calvo Sotelo esté pendiente de su incorporación a la OTAN, así como del inicio de negociaciones con Gran Bretaña sobre el futuro de Gibraltar (previstas para el 20 de abril) y de la renovación de los acuerdos bilaterales con Estados Unidos (prevista para el mes de mayo, pero que no se firma hasta el 2 de julio, tras complejas negociaciones) (Marquina Barrio, 1986: 909-931)

3.- España mantiene con América Latina una políticałque la diferencia de los países de su entorno europeo. Así, la diplomacia post-franquista caracteriza las relaciones de España con dichos países en términos de "hermandad" o de "relaciones de familia" (Oreja, 1976: 17). Lo que, desde el punto de vista de las diplomacias europeas, constituye una especificidad española.

La reacción española frente a la guerra de las Malvinas se caracteriza, como veremos, por su ambigüedad. Dicha reacción es un producto de las circunstancias del momento (crisis del Gobierno y proceso de ingreso en la OTAN, entre otras) pero, al mismo tiempo, refleja uno de los dilemas básicos de la política exterior de la España democráticả: la articulación de sus relaciones con América Latina, por una parte, y con la Europa comunitaria, por la otra. De hecho, dicha articulación es el factor determinante en las relaciones hispano-argentinas, una vez producido el ingreso de España en la Comunidad Europea (tema que vamos a abordar, en un apartado final, en tanto que escenario de la posición española respecto del conflicto de las Malvinas, una vez acabada la guerra).

\section{I.- Foreign policy making en la España democrática.}

El período de estudio que nos ocupa coincide con la creación de un estilo de foreign policy making por parte de la España democrática. En realidad, desde la promulgación de la Constitución en 1978 se puede hablar de la existencia de un marco institucional democrático para la elaboración de la política exterior. Ahora bien, en lo que respecta a orientación y a definición de objetivos, algunos autores (Aldecoa, 1984) consideran que la diplomacia de la España democrática tiene sus orígenes en el programa diseñado por el Ministro de Asuntos Exteriores Fernando María Castiella (1957-1969) quien, consiguiendo una cierta autonomía del servicio exterior con respecto a Franco, llevó a cabo una política contraria a la satelización de 
España por los Estados Unidos y en busca de un "espacio propio" gracias a una política activa en los foros internacionales. El período Castiella, que crea un estilo diplomático persistente en la democracia, es una fase más en la diplomacia franquista que en su conjunto se caracterizó por la ambigüedad, el pragmatismo y el equilibrio (Halstead, 1980). Así, por ejemplo, el estilo Castiella (reivindicativo y activo) se vio én los últimos años de la vida de Franco sustituido por un estilo mucho más discreto (próximo a Washington) y con orientación económica.

En realidad, la práctica y los hábitos de trabajo de la España democrática en materia internacional se han perfilado esencialmente a partir de la llegada del PSOE al poder en 1982 y, en concreto, bajo la dirección política del Presidente González.

La Constitución española de 1978 ha merecido, entre los analistas españoles (Remiro Brotons, 1984; Mesa, 1985), críticas negativas, siendo calificada de poco innovadora y de limitada en lo que respecta a la democratización de la política exterior y a la introducción de principios internacionalistas. Desde el punto de vista de los principios y desde el punto de vista institucional, la Constitución española es claramente conservadora en relación con la dimensión exterior del Estado.

La elaboración de la política exterior se divide, según la Constitución, entre el Rey, encargado de representar al Estado español en las relaciones internacionales (art. 56); el Gobierno, encargado de dirigir la política exterior (art.97) y las Cortes, en su función genérica de control del Gobierno (art. 66).

La figura representativa del Rey de España en las relaciones internacionales se ha convertido en una baza importante para la diplomacia española. De tal manera, que a través de Juan Carlos I se ha prestigiado la imagen internacional del'país. El papel del Rey es especialmente destacado en las relaciones de España con el mundo árabe y con América Latina. En este último caso, como ya veremos, la propia Constitución destaca el papel representativo del monarca.

La dirección de la política exterior por parte del Gobierno como colectivo supone, de hecho, la dirección de dicha política por parte del Presidente del Gobierno. La voluntad de los constituyentes chocaba así contra un sector de opinión, encabezado por el Ministro de Asuntos Exteriores del momento, Marcelino Oreja, que había defendido la necesidad de convertir al Ministerio de Asuntos Exteriores en "el principal responsable del servicio exterior del Estado y 
de la cooperación internacional" (Oreja, 1978: 57). Contrariamente, la Constitución convierte de manera implícita al Presidente del Gobierno en el director de la política exterior.

La práctica posterior y los hábitos de trabajo han reforzado el presidencialismo latente en la Constitución. Así, la personalidad y el liderazgo, primero de Adolfo Suárez (1976-1980), pero después, de manera decisiva e importante de Felipe González (1982- ), permiten hablar de "sistema presidencialista" (Saba, 1986: 28) en lo que respecta a la elaboración de la política exterior. La única excepción la constituye el corto período, como Presidente del Gobierno, de Leopoldo Calvo Sotelo (1980-1982). De hecho, su etapa presidencial ha sido calificada de "encogimiento" en la política exterior española (Aldecoa, 1989: 72), dado que Calvo Sotelo fue un político poco activo en materia internacional, si exceptuamos su decidida voluntad de integrar a España en la OTAN.

Felipe González ha demostrado claramente su voluntad de asumir la dirección de la política exterior. De ahíla creación por parte del Presidente de su propio equipo de asesores en relaciones internacionales, calificado por Roberto Mesa (1985: 158) de Ministerio paralelo de Asuntos Exteriores. Otro dato que demuestra el interés del Presidente por la política exterior son sus viajes oficiales al extranjero. Entre 1982 y 1991, Felipe González había realizado 108 visitas oficiales a países extranjeros (frente a 30 visitas del mismo tipo en España).

La Constitución, que ha introducido una "democratización limitada" (Remiro, 1984: 23) en materia de política exterior, ofrece a las Cortes $^{3}$ un papel similar al que otorgan la mayoría de las constituciones de Europa occidental a sus respectivos parlamentos. Así, las Cortes participan en la formación y en la denuncia de determinados tratados internacionales, y autorizan al Rey a declarar la guerra y a hacer la paz. De ahí, las críticas al carácter elitista y poco democrático de la Constitución en materia exterior. Remiro Brotons (1984: 23) recuerda, a ese respecto, propuestas más progresistas que surgieron durante el período constituyente -ampliar el papel de las Cortes, regular de manera generosa la utilización del referéndum, introducir la iniciativa popular-y, que finalmente se abandonaron en favor de una concepción más tradicional y conservadora.

\footnotetext{
${ }^{3}$ Las Cortes, o Parlamento español, están formadas por dos Cámaras, Congreso y Senado.
} 
El control de la política gubernamental por parte de los parlamentarios -en el marco, básicamente, de las Comisiones de Asuntos Extenores de ambas cámaras- ha sido más reducido que en las materias de política interna. En primer lugar, porque hasta 1982 se pactó una política de consenso entre las fuerzas políticas en materia de política exterior con la intención de favorecer la transición política interna (Barbé, 1990a). Desde 1982, y a causa del ingreso español en la OTAN contra la voluntad de importantes grupos parlamentarios (socialistas y comunistas), acaba formalmente el consenso en materia de política exterior. Sin embargo, una vez entrados en la década socialista (a partir de las elecciones de octubre de 1982), la mayoría absoluta del PSOE en las Cortes, por una parte, y el apoyo de la oposición, por otra, en temas decisivos (política en la Comunidad Económica Europea, participación en la guerra del Golfo) ha hecho que el legislativo mantuviera, de forma global, una política claramente convergente con la del Gobierno de Felipe González en materia internacional. De ahí que, en buena medida, se pueda hablar de política de Estado en relación con la política exterior de España en el período que nos ocupa (1982-1992), excepción hecha del confuso tema de la permanencia de España en la OTAN.

\section{II.- Posición española ante la guerra de las Malvinas: descolonización y condena del uso de la fuerza.}

La guerra de las Malvinas (2 abril/15 junio 1982) coincide con un momento sensible para la política española, tanto en el terreno interno como en el internacional. A nivel interno, España pasa por una etapa decisiva para la consolidación de la transición democrática (juicio a los golpistas del 23 de febrero y disolución del partido en el Gobierno). A nivel exterior, el Gobierno está ocupado con los temas más sensibles de su agenda (OTAN, Gibraltar y relaciones con Estados Unidos). Lo que lleva a Mujal León a escribir que el Gobierno de Calvo Sotelo "centró su atención en los temas internos, perspectiva que dominó incluso la decisión de Calvo Sotelo sobre el ingreso en la OTAN. Consecuentemente, España perdió todo interés en América Latina, una tendencia reforzada por los acontecimientos de Nicaragua a partir de 1979 y por la guerra de las Malvinas/Falklands de 1982" (1986: 378). De manera metafórica, se puede decir que la guerra es 
"inoportuna" desde el punto de vista de los intereses del Gobierno español.

En efecto, la descripción y el posterior análisis de la postura española durante la guerra de las Malvinas nos permitirá verificar la "inoportunidad" de la misma para los intereses españoles en 1982, así como extraer lecciones de orden general sobre las opciones españolas en materia internacional.

Para comenzar, se expone de manera descriptiva la actitud del Gobierno, del Rey y de los partidos políticos y otros grupos sociales en relación con la guerra.

Ante la ocupación argentina de las Islas, la reacción del Gobierno español fue rápida, ya que el mismo 2 de abril el Consejo de Ministros emitió una nota oficial que asentaba la posición del Gobierno. Dicha nota contenía cuatro ideas básicas: 1.- la defensa de la descolonización de las Islas en favor de la integridad territorial argentina; 2.- la prolongación de las situaciones coloniales es fuente de tensiones y conflictos; 3.- la oposición al uso de la fuerza; y 4.- la solución de los conflictos por vía pacífica, a través de Naciones Unidas y de su Secretario General.

Los principios defendidos en la nota del día 2 de abril -descolonización y condena del uso de la fuerza-constituyen la base de la posición española a lo largo de toda la guerra. En base a dicha posición, el representante español ${ }^{4}$ en el Consejo de Seguridad de Naciones Unidas se abstiene el 3 de abril en la votación de la Resolución 502 que "exige el cese inmediato de las hostilidades (...), la retirada de las fuerzas argentinas (...) y la búsqueda de una solución diplomática". El representante español, Jaime de Piniés, expone la posición de su país contraria al uso de la fuerza. Sin embargo, justifica su abstención en base a la no mención en la resolución del problema de fondo-la descolonización-, recordando en dicho sentido el apoyo del Gobierno español a las reivindicaciones territoriales de Argentina (Fernández Sánchez, 1984: 933).

El apoyo español a las reivindicaciones territoriales de Argentina en las Malvinas es destacado por el propio Ministro de Asuntos Exteriores español, quien recuerda la continuidad de la política española en dicho tema (Pérez Llorca 1982: 125), ya que España había votado tradicionalmente en la Asamblea General de Naciones

\footnotetext{
${ }^{4}$ En 1982, España ocupaba un puesto de miembro no-permanente en el Consejo de Seguridad.
} 
Unidas (diciembre 1965, diciembre 1973, diciembre 1976) en favor de la descolonización del archipiélago (Sțavridis, 1992: 4).

El principio de la descolonización se convierte así en el factor diferenciador de la política española en la cuestión de las Malvinas respecto de la política adoptada por los países de su entorno geopolítico. La actitud de España en relación con la posición del Consejo de Europa, de la Comunidad Europea y de la OTAN es ilustrativa.

El Comité de Ministros del Consejo de Europa elabora una resolución (el 7 de abril), a partir de los términos recogidos en la resolución 502 del Consejo de Seguridad. España es el único país que se abstiene en la votación, en base al mismo argumento utilizado en Naciones Unidas. ${ }^{5}$

La Comunidad Europea acuerda (el 14 de abril) un embargo económico contra Argentina (Edwards, 1984). Una vez informado el Gobierno español, éste rechaza su participación en el embargo entendiendo, según el Ministro español, "que se trata de un grave error político de la Comunidad, que no ha valorado suficientemente la profundidad de la reivindicación nacional argentina" (Pérez Llorca, 1982: 126). De hecho, el Gobierno español se suma durante una reunión del GATT (8 de mayo) a una propuesta del grupo iberoamericano para revocar el embargo impuesto a Argentina por la Comunidad, Estados Unidos y Australia, por entender que el mismo infringe las normas del GATT.

El ingreso de España en la OTAN, a partir del mes de junio comporta un foro occidental más en el que España debe destacar su diferencia. El apoyo unánime de los quince miembros de la OTAN a Gran Bretaña en su enfrentamiento con Argentina se ve roto con las primeras manifestáciones oficiales del Ministro Pérez Llorca (el-4 de junio) en defensa de la integridad territorial argentina. ${ }^{6}$ Durante el Consejo Atlántico, celebrado en Bonn (10 de junio), el Presidente Calvo Sotelo aprovechó para mencionar "la grave grieta" que está abriendo el conflicto y para recordar "la vocación iberoamericana" de España, sin aludir directamente al apoyo de sụ Gobierno a la descolonización de las Islas.

\footnotetext{
${ }^{5}$ El Ministro español no asistió a la mencionada reunión, para evitar un posible encuentro con su homólogo británico.

${ }^{6} \mathrm{El}$ Ministro Pérez Llorca había aducido motivos de trabajo para no asistir como observador a una reunión preparatoria del Consejo Atlántico, celebrada en Luxemburgo el 17 de mayo. De esta manera, el Ministro evitaba abordar la cuestión de las Malvinas en la espera de una rápida solución del conflicto antes del ingreso efectivo de España en la OTAN.
} 
La posición del Gobierno español, que le sitúa en un terreno ambiguo y en cualquier caso diferente del de los países de su entorno geopolítico, ${ }^{7}$ da muestras de mayor activismo a partir del momento en que la escalada del conflicto (bombardeos, hundimiento de buques) aumenta espectacularmente el número de víctimas. Así, a partir del 1 de mayo en que comienzan los bombardeos británicos sobre las Islas, el Gobierno español da muestras de mayor actividad. Dicho activismo se traduce en: 1.- ofertas de mediación (el 3 de mayo, Calvo Sotelo anuncia que se había ofrecido como mediador a primeros de abril; el mismo día Pérez Llorca ofrece nuevamente a Pérez de Cuéllar la mediación española); 2.- apoyo a acciones iberoamericanas (la oferta de mediación de Belaunde Terry [Bendahmaney Mc Donald, 1986] o la presentación al Consejo de Seguridad junto con Panamá, el 2 de junio, de un proyecto de resolución que solicita el alto el fuego y que es rechazado dos días después con veto británico); 3.- gestiones bilaterales (Pérez Llorca solicita de Haig, durante su visita a Washington el 5 de mayo para negociar los acuerdos bilaterales, que los Estados Unidos actúen en favor del cese de las acciones armadas); 4.- el apoyo continuado a la actividad negociadora del Secretario General de Naciones Unidas.

La posición adoptada por el Gobierno español y las gestiones realizadas -ya en la fase más dura de la guerra-revelan, a partir de las palabras de Pérez Llorca, dos objetivos esenciales. Según Pérez Llorca: "Yo creo que España, que ha mantenido siempre una posición independiente, la que tenía que ser la suya en este tema, clara e inequívoca, pero serena, ha contribuido en la medida de lo posible, a que no haya una escisión trágica, en definitiva, entre el sentimiento europeo y el sentimiento iberoamericano" (Pérez Llorca, 1982: 129). Es decir, por un lado, la voluntad del Gobierno español de mostrarse independiente a la hora de elaborar su política exterior y, por otro lado, la voluntad de jugar el papel de puente entre Europa e Iberoamérica.

Una vez expuestas las líneas generales de la posición gubernamental, es preciso hacer una mención a la actividad del Rey de España en relación con la guerra. Más si tenemos en cuenta que la misma afecta a un país iberoamericano y según la Constitución

\footnotetext{
${ }^{7}$ Leo Tindemans, Presidente de turno de la Comunidad Europea declara el 3 de mayo que la posición española es "especial y delicada". La postura de Irlanda y de Italia, reacias a imponer las sanciones a Argentina, genera satisfacción en España, siendo así menos especial la posición española.
} 
E. Barbé / Entre Europa y América Latina: la diplomacia ..

española de 1978 el Rey "asume la más alta representación del Estado español en las Relaciones Internacionales, especialmente con las naciones de su comunidad histórica" (art. 56). Recurso que hace mención, de manera implícita, a los países americanos de habla hispana.

El argumento histórico de la vinculación española con Iberoamérica está presente en la carta enviada por el Rey de España al Secretario General de Naciones Unidas (el 5 de mayo), solicitando un alto el fuego y ofreciéndose "para contribuir a la consecución de la paz y de la justicia". La oferta de mediación del monarca, sin resonancia alguna en Europa, es bien acogida en Iberoamérica.

La acción del monarca español, que tras el mensaje al Secretario General se concluye con otro mensaje, en este caso al Presidente Reagan (el 20 de mayo) solicitándole un esfuerzo en el terreno diplomático, se articula claramente alrededor del papel de España como país transeuropeo, proyectado más allá del continente y como miembro de una comunidad de naciones hispánicas. Esta reflexión impregna el discurso del monarca durante el acto de entrega del Premio Carlomagno en Aquisgrán (el 20 de mayo).

La acción del monarca, limitada diplomáticamente a los mensajes mencionados, tiene importancia en lo que respecta a su propia figura en el marco interno (prestigio como defensor del sistema democrático) y en el marco iberoamericano. De hecho, la actuación del Rey, aprobada de manera unánime por todas las fuerzas políticas y sociales, hay que ubicarla en el contexto interno de la consolidación de la democracia y no en el debate genérico sobre el papel de España en la guerra.

\section{III.- Los grandes debates políticos durante la guerra.}

La posición gubernamental y las gestiones del monarca a lo largo de la guerra se enmarcan en un estado de opinión, que describiremos de manera exhaustiva a través de la posición de los partidos políticos y, de manera puntual, a través de referencias a otras fuerzas sociales, a la prensa y a la opinión pública.

El papel más destacado recae en los partidos políticos, que encuentran en la guerra de las Malvinas un pretexto, oportuno, para someter la política exterior del Gobierno a debate. No hay que 
olvidar que en abril de 1982 las fuerzas parlamentarias ya no están ligadas por la política de consenso en materia exterior (rota por la decisión gubernamental de entrar en la OTAN, con el voto en contra de socialistas y comunistas). De ahí, por tanto, que la guerra en el Atlántico Sur se convierta en una ocasión única para debatir el conjunto de la acción exterior del Estado con una agenda muy cargada: las negociaciones sobre Gibraltar, el ingreso en la OTAN, la renovación de los acuerdos con Estados Unidos y, como telón de fondo, el ingreso del país en las Comunidades Europeas.

Las fuerzas parlamentarias intentaron, sin éxito, forzar la convocatoria de un pleno del Congreso, con votación de una resolución. El Gobierno consiguió llevar el tema a debate, sólo en la Comisión de Asuntos Exteriores y sin voto. La reunión tuvo lugar el 11 de mayo y en ella los diferentes grupos expusieron su opinión sobre el comportamiento del Gobierno frente a la guerra y las consecuencias del mismo para las relaciones exteriores de España en su conjunto. Las intervenciones parlamentarias en dicha sesión abordaron todos los temas que a nivel de opinión pública y de fuerzas sociales había recogido la prensa. De ahí que la mencionada sesión permita establecer un esquema recapitulativo de grandes temas. A efectos de análisis los hemos dividido en cinco grandes temas, presentando algunos de ellos bajo la forma de cleavages. Son los siguientes:

1.- La guerra en las Malvinas como tema autónomo o, bien, como tema que afecta al conjunto de la agenda diplomática española,

2.- La aproximación a la guerra, de manera prioritaria, en términos de principio de la descolonización o, bien, a partir de la condena del uso de la fuerza,

3.- La aproximación a la guerra, de manera prioritaria, a partir del carácter dictatorial del régimen argentino o, bien, descartando toda referencia al eje dictadura/democracia en el conflicto,

4.- La guerra ha demostrado la capacidad o, por el contrario, la incapacidad del Gobierno español para elaborar una política exterior independiente,

5.- La guerra ha planteado hasta qué punto las relaciones con Iberoamérica constituyen la opción prioritaria de España en el marco internacional.

Sin ánimo exhaustivo, se abordan sucesivamente los cinco grandes temas del debate para ver como se posicionan en cada uno de ellos las grandes fuerzas políticas y, puntualmente, otras fuerzas sociales y los medios de comunicación. 
1.- La Unión de Centro Democrático (UCD), como partido gobernante, es la encargada de defender la ortodoxia gubernamental, en el sentido de que la guerra es un tema autónomo, al margen de la agenda global de la política exterior española. De ahí declaraciones de algunos diputados de la UCD: "Tenemos el firme convencimiento de que no hay que mezclar los temas específicamente españoles con el conflicto malvino". (Muñoz Peirats, 1982: 5), o bien, "las Malvinas no valían una guerra. Tampoco valen una fisura en nuestra proyección exterior" (Rupérez, 1982: 5).

En contraste con la posición de la UCD, hay que mencionar que todos los partidos políticos y el conjunto de las fuerzas sociales, si atendemos al análisis de la prensa española (Stavridis, 1992: 16-26), sostienen la idea de que la guerra afecta directamente a los intereses españoles. Comenzando por la cuestión de Gibraltar, tema que aúna todas las opiniones. No hay que olvidar que a nivel de opinión pública española la cuestión gibraltareña es altamente sensible. ${ }^{8}$ Si la vinculación entre las Malvinas y Gibraltar se puede definir como una opción de mínimos, aceptada ampliamente, también podemos hablar de una opción de máximos. Es lo que hará el PSOE, al vincular la guerra con todos los temas sensibles de la agenda española: "La crisis de las Malvinas en su planteamiento actual y en su evolución posible tiene incidencia sobre: a) la viabilidad y ritmo del procedimiento elegido para obtener la reintegración de Gibraltar; b) aspectos importantes del supuesto plan del Gobierno respecto a la forma de integración en la OTAN; c) el clima que rodea a nuestras negociaciones para adherirnos a las Comunidades Europeas; d) la necesidad absoluta de armonizar nuestros intereses europeos y nuestra vocación, vínculos y comprensión de Latinoamérica" (Morán, 1982: 13).

2.- La UCD defiende la postura gubernamental consistente, como sabemos, en defender simultáneamente el principio de la descolonización y la condena del uso de la fuerza. Entre los partidos políticos hallamos opciones diametralmente opuestas. De un lado, la

\footnotetext{
${ }^{8}$ Las encuestas de opinión pública muestran el interés que los españoles conceden al tema de Gibraltar. Así, en 1979 (diciembre) el Centro de Investigaciones Sociológicas elabora un estudio en el que 2.022 personas han de señalar en una lista de nueve temas de la política exterior española, los tres que consideran más importantes, con el resultado global de que el más importante es la entrada en el Mercado Común (50\%), seguido por la recuperación de Gibraltary las relaciones con Iberoamérica. En ambos casos, con el 32\%. Además, la respuesta en el caso de la recuperación de Gibraltar es muy homogénea independientemente de la ideología del encuestado: Izquierda (36\%), Centro (37\%), Derecha (39\%) y Extrema Derecha ( $37 \%$ ), a excepción de la Extrema Izquierda (13\%). Véase "Estudio sobre relaciones exteriores. Diciembre 1979", Revista Española de Investigaciones Sociológicas, vol. 22, 1983, pp. 208-209.
} 
opción de Alianza Popular (AP) que considera, por encima de todo, el derecho a la descolonización. En ese sentido, los dirigentes populares critican de manera dura el colonialismo decimonónico de Gran Bretaña, recordando el fin de la era de los colonialismos.

Frente a la clara primacía del principio descolonizador; adoptada por AP, el PSOE (junto a comunistas, Partido Nacionalista Vasco y Minoría Catalana) destacan, de entrada, su condena al uso de la fuerza, identificando a Argentina como país agresor. Lo que no impide que, especialmente en las filas comunistas, se critique duramente la "brutalidad británica", en palabras de Santiago Carrillo.

En cualquier caso, el PSOE condena la actitud del Gobierno en Naciones Unidas por su abstención en la Resolución 502. En ese sentido, los socialistas consideran que la actitud española es peligrosa a nivel interno (patriotismo español que desborde en fuerza armada en el caso de Gibraltar) y de cara a Marruecos (no condenar plenamente el uso de la fuerza abre una puerta a futuras acciones marroquíes en Ceuta y Melilla). ${ }^{9}$ Asimismo, los socialistas critican el hecho de que el Gobierno no mantenga posiciones equidistantes, al criticar más duramente el uso de la fuerza por parte británica que por parte argentína, en referencia al comunicado de la Oficina de Información Diplomática (1 de mayo) que condena la escalada del conflicto por parte dé los británicos. Según los socialistas, dicha actitud descalifica a España como posible mediador.

3.- El carácter dictatorial del régimen argentino también se convierte en otro punto de diferenciación entre los partidos políticos y en el seno de la sociedad española. El Gobierno, por su parte, no había valorado el carácter dictatorial del régimen argentino como un factor destacable en el conflicto. Según el Ministro de Asuntos Exteriores: "El problema de las Malvinas ha dejado de ser un conflicto local para convertirse en una posible confrontación generalizada entre dos pueblos. Dos pueblos que, con independencia de sus sistemas políticos, pertenecen al mismo mundo" (Pérez Llorca, 1982: 124). De hecho, la posición que infravalora la alternativa dictadura/democracia, en relación con la guerra, domina en las filas de la UCD.

Este tema enfrenta diametralmente a las fuerzas políticas. En un extremo, AP considera que el carácter del régimen político no es un tema importante cuando existe una auténtica reivindicación na-

\footnotetext{
${ }^{9}$ Ceuta y Melilla son dos ciudades españolas, ubicadas sobre la costa africana y enclavadas en territorio marroquí.
} 
cional, señalando que incluso los Montoneros se han situado junto a la Junta Militar en el tema de la recuperación de las Islas (Kirpatrick, 1982: 15).

Frente a la actitud nacionalista de AP, el PSOE, el PC y el PNV destacan el carácter dictatorial del régimen y cómo la invasión de las Islas constituye una fuite en avant para la Junta Militar. Esta cuestión incide de manera muy directa en la propia réalidad española, dado el proceso de consolidación de la democracia que vive el país. Algunos medios de información-como El País y Cambio 16-otorgan un papel muy destacado en su análisis al carácter respectivamente dictatorial (Argentina) y democrático (Gran Bretaña) de los contendientes. Ahora bien, la mayor parte de la prensa española ignora o apenas destaca el factor de la dictadura argentina en su información.

Hay que apuntar aquí, también, la formación en Madrid de un "Movimiento contra la guerra en el Atlántico Sur", integrado por 500 argentinos exiliados en España. Dicho Movimiento basa su campaña en la idea de que la única soberanía posible pasa por la caída de la dictadura militar.

4.- La capacidad del Gobierno español para elaborar una política exterior independiente es un tema central en el debate. Como hemos visto, el Gobierno insiste en manifestar su independencia de criterio. Posición en la que se ve apoyado por la UCD. En ese sentido, Javier Rupérez, portavoz de la UCD en la Comisión de Asuntos Exteriores, llegó a manifestar durante la sesión de dicha Comisión (el 11 de mayo) que la actitud del Gobierno de Calvo Sotelo durante la guerra no era ni pro-argentina, ni pro-británica, sino pro-española.

En sentido contrario a lo defendido por la UCD; PSOE, PC y Grupo Mixto ${ }^{10}$ consideran, de manera unánime, que la posición gubernamental ha sido ambigua y de bajo perfil, a causa del escaso margen de autonomía que le queda a España una vez decidido el ingreso en la OTAN. En ese sentido, destacados socialistas reclaman para España el título de potencia media y, como tal, la posibilidad de reservarse un área de decisiones autónomas al margen de los intereses globales (Morán, 1982: 13).

\footnotetext{
${ }^{10} \mathrm{El} \mathrm{Grupo} \mathrm{Mixto} \mathrm{está} \mathrm{formado} \mathrm{por} \mathrm{grupos} \mathrm{políticos} \mathrm{que} \mathrm{no} \mathrm{disponen} \mathrm{de} \mathrm{suficientes} \mathrm{escaños}$ para crear grupo parlamentario propio. En 1982, Francisco Fernánđez Ordóñez (antes Ministro de la UCD y, a partir de 1985, Ministro de Asuntos Exteriores con el PSOE) formaba parte de dicho Grupo.
} 
Se trata, como vemos, de un tema que la guerra ha permitido replantear contra la voluntad del Gobierno que ya había ahogado el debate sobre la OTAN en el Parlamento. Debate que la opinión pública ve con buenos ojos, si tenemos en cuenta que en 1981, una vez iniciados los trámite de ingreso en la Alianza, el 50\% de los españoles se mostraban contrarios a dicho ingreso (frente a un $20 \%$ favorable) (Equipo de Sociología Electoral, 1986: 207).

Algunos parlamentarios, como el futuro Ministro de Asuntos Exteriores (Fernández Ordóñez), utilizan la experiencia de las Malvinas para replantear las condiciones del ingreso español en la OTAN, condicionándolo a la obtención de garantías por parte de la Alianza sobre Gibraltar, Ceuta y Melilla.

5.- Las relaciones de España con América Latina constituyen un tema presente en las múltiples perspectivas antes abordadas. Ninguna fuerza política, ni la sociedad en general, niega la existencia de relaciones especiales entre España y los países de América Latina; traducidas a nivel de televisión pública en una información claramente pro-argentina (Stavridis, 1992), criticada por otros medios de comunicación (un editorial de El País del 16 de junio acusa a Televisión Española de desvirtuar la realidad a lo largo de la guerra en favor de Argentina). Ahora bien, estas relaciones se expresan en algunos casos a través de una retórica propia del franquismo. Es el caso de AP o del Partido Andalucista, ${ }^{11}$ que abordan las relaciones en términos de familia (nuestros hermanos argentinos) y de honor (traición). En esta dimensión se ubica una declaración de la "Federación de Sociedades Hispanas" (formada básicamente por centros regionales) en la que se indica que el pueblo español está con Argentina y se informa del ofrecimiento de 60 oficiales españoles en la embajada argentina en Madrid para luchar en el Atlántico Sur.

La UCD, por su parte, ha querido defender la posición del Gobierno decidida a ampliar la dimensión histórica especial entre España y los países de América Latĩna con el papel de puente entre ese continente y Europa. Se puede decir que esta última función, en absoluto desempeñada por el Gobierno español durante la guerra, anima la creación en Madrid de una "Asociación Hispanoamericana para la Integración, el Desarrollo y la Democracia Avanzada", for-

\footnotetext{
${ }^{11} \mathrm{La}$ actitud ante las relaciones con América Latina no es homogénea en toda España. Así, mientras en Andalucía 32\% de la población cree que dichas relaciones son uno de los tres temas más importantes de la política exterior española, en Cataluña sólo lo cree así el 11\%. Véase "Estudio sobre relaciones exteriores. Diciembre 1979", op. cit.
} 
mada por intelectuales españoles y latinoamericanos que tiene como objetivo la renovación de las relaciones entre Europa y América Latina.

Ahora bien, en el seno de la UCD se detectan diferencias sobre este particular, entre los seguidores del ex-Presidente Adolfo Suárez y el Gobierno de Calvo Sotelo. No es extraño, si tenemos en cuenta que la presidencia de Suárez se había caracterizado en materia internacional por un cierto "tercermundismo", en el que destacaban las relaciones con América Latina (Armero, 1989: 125), a diferencia de la presidencia Calvo Sotelo que había optado de pleno por la inserción de España en el mundo europeo-occidental.

La opción latinoamericana de socialistas y de comunistas se inscribe en un contexto menos histórico y más político, que combina múltiples elementos, tanto del pasado reciente (antiamericanismo, ${ }^{12}$ en referencia a los Estados Unidos, tercermundismo) corno del presente (la defensa de una esfera autónoma para la política exterior de la España democrática en el marco europeo y la defensa de la democracia en el continente americano) de ambos partidos.

\section{IV.- 1982: el triángulo de la diplomacia española.}

Una vez descrita la posición del Gobierno y de las fuerzas políticas españolas durante la guerra, podemos revisar la hipótesis de partida sobre la inoportunidad de la guerra para el desarrollo de la agenda diplomática española.

Los analistas han calificado la actitud del Gobierno español durante la guerra de ambivalente (Stavridis, 1992: 2), salomónica y conducente a la parálisis (Mujal León, 1986: 360). En realidad, la guerra entre británicos y argentinos es inoportuna porque sitúa al Gobierno español frente a una evidencia molesta: la agenda prioritaria en materia internacional es irrealizable de manera conjunta. No es de extrañar, por tanto, que los protagonistas del momento, como el propio Calvo Sotelo (1990) o su Ministro del Interior, Rodolfo Martín Villa (1984), hayan obviado en sus memorias toda referencia

.12 Un dato del antiamericanismo existerite entre la sociedad española nos lo dan las encuestas sobre percepción de amenaza. En 1980, el 12\% de los españoles creían que la seguridad nacional estaba amenazada. Entre ellos, el $29 \%$ pensaba que la amenaza más importante eran los Estados Unidos, por delante de la Unión Soviética, un $20 \%$, y de Marruecos, un $11 \%$. Véase "Estudio CIS", Centro de Investigaciones Sociológicas, E. 1207 (junio 1980). 
a la guerra o lo hayan hecho, como en el caso de Javier Rupérez (1986: 203), por aquel entonces diputado de la UCD y embajador español en la OTAN, de manera indirecta, como elemento perturbador del tema gibraltareño.

Volviendo a la agenda diplomática del Gobierno español en 1982 se puede apuntar, en términos metafóricos, que es algo así como un triángulo equilátero. Un triángulo que se presenta con tres vértices idénticos (idéntica separación entre los mismos) para la política exterior del Gobierno español. Los tres vértices están ocupados por: 1.- la descolonización de Gibraltar, 2.- el ingreso de España en las Comunidades Europeas (no se introduce aquí el tema OTAN porque ya está finalizado desde el punto de vista de la negociación multilateral) y 3.- el mantenimiento por parte de España de relaciones privilegiadas con América Latina.

El primer vértice está ocupado por la reivindicación de Gibraltar. Esta reivindicación -enérgicamente defendida en el marco de Naciones Unidas por Fernando M. Castiella, Ministro de Asuntos Exteriores durante el franquismo y autor de un famoso libro sobre el tema (1941)- había ocupado un lugar destacado en la agenda de la diplomacia franquista. La transición a la democracia no disminuye la importancia del tema. En este sentido, Roberto Mesa apunta que "Gibraltar no es una reivindicación franquista, rii una empresa quijotesca del Ministro Castiella: la recuperación de Gibraltar es una exigencia de todo el pueblo español, para así finalizar el proceso de integridad territorial" (1988: 46). ${ }^{13}$ Ahora bien, la transición democrática resitúa la reivindicación de Gibraltar dentro de un espacio político diferente: la europeización de España a través de su ingreso en foros multilaterales formados por países democráticos.

El tema de Gibraltar conoció un avance importante a partir de 1980, cuando los gobiernos de Madrid y Londres decidieron en Lisboa (10 de abril) la apertura de negociaciones para superar sus diferencias sobre Gibraltar. $\mathrm{El}$ acuerdo de Lisboa dio un paso adelante el 8 de enero de 1982, con motivo de una visita de Calvo Sotelo a Margaret Thatcher. En la misma, se decidió que el 20 de abril se iban a iniciar negociaciones y que el Gobierno español abriría al

\footnotetext{
${ }^{13}$ Según una encuesta realizada en 1991 (a 1.000 entrevistados), el 64,2\% de los españoles considera que Gibraltar constituye un obstáculo para las relaciones hispano-británicas. A la hora de abordar la mejor solución para Gibraltar, el 93,6\% de los españoles cree que es el retorno de la soberanía a España. Véase Salustiano del Campo, Informe Incipe 1991. La opinión pública españoia y la polftica intemacional, (Madrid: Tecnos, 1991), pp. 62-63.
} 
mismo tiempo la verja que aislaba desde los años sesenta a los gibraltareños. A su vez, Thatcher manifestó el apoyo de su país âl ingreso de España en la Comunidad Europea y en la OTAN.

La guerra de las Malvinas estalló pocos días antes del inicio de las negociaciones hispano-británicas sobre Gibraltar. No es extraño que la primera reacción de Calvo Sotelo (el 3 de abril) fuera la de considerar que el caso de las Islas es distinto y distante del de Gibraltar, en un intento de salvar las negociaciones hispano-británicas. La misma decisión de abstenerse en la votación de la Resolución 502 del Consejo de Seguridad es interpretada por algunos analistas como una manera de salvar el proceso negociador sobre Gibraltar (Morris y Haigh, 1992: 110). Sin embargo, el 9 de abril se decidió el aplazamiento de las negociaciones hasta el 25 de junio a petición española, ante la evidencia de que el momento era inapropiado. En realidad, la opinión dominante en España era que la guerra en el Atlántico Sur había retrasado la solución del tema de Gibraltar. Así una editorial de El País (31 de mayo) indicaba que "resulta difícil suponer que Londres se vaya a desprender tan fácilmente de Gibraltar después de la sangría humana del Atlántico Sur y en medio de un ambiente de exacerbado nacionalismo en Gran Bretaña". La parálisis del Gobierno Calvo Sotelo por motivos internos y el mal momentum (postguerra) llevan al aplazamiento de las negociaciones hasta la llegada de los socialistas al poder. Fernando Morán, nuevo Ministro de Asuntos Exteriores y diplomático formado en las ideas de Fernando M. Castiella, será el encargado de abrir, por razones humanitarias, la verja de Gibraltar a los peatones (el 15 de diciembre de 1982) y de iniciar las conversaciones con los británicos que llevarán al comunicado de Bruselas (noviembre de 1984), por el que se restablecen plenamente las relaciones entre Gibraltar y España y en el que, por primera vez, Londres se declara dispuesta a negociar la cuestión de la soberanía.

Ahora bien, la actuación española en el tema de Gibraltar ha estado mediatizada por el segundo vértice de su política exterior: el ingreso en las Comunidades Europeas. Es decir, el Gobierno español ha de optar en 1982 entre unas relaciones cooperativas con Gran Bretaña, básicas para el ingreso en la Comunidad, o unas relaciones seguramente conflictivas, centradas en la reclamación de la soberanía española sobre Gibraltar. El propio Ministro Morán reconocerá, después de su cese, que en muchos momentos se había "sentido solo" 
(Morán, 1986: 20), en referencia al desinterés del Gobierno por su batalla en el tema de Gibraltar.

En el tema comunitario era evidente en 1982 que España necesitaba el apoyo británico (Groom, 1991: 61), dada la actitud francesa. Hay que recordar aquí que Giscard d'Estaing había congelado las negociaciones para la adhesión española en 1980 (Preston y Smith, 1985: 150) y que Mitterrand durante una visita a España, pocos días después del final de la guerra de las Malvinas (el 22 de junio), había manifestado reservas ante el ingreso español en la Comunidad Europea.

Durante la guerra (el 9 de junio), Margaret Thatcher se había manifestado públicamente en favor del ingreso de España en la Comunidad, reforzando de esta manera la baza negociadora de los británicos en Gibraltar. En este último sentido, Fernando Morán ha escrito que la Primera Ministra británica le manifestó durante su primer encuentro negociador sobre Gibraltar (el 16 de marzo de 1983) que la actitud española sobre este tema (reivindicar la soberanía) iba en detrimento de su solicitud de ingreso en la Comunidad (Morán, 1990: 110).

La vinculación entre ambos temas -que lleva al Gobierno español a priorizar el segundo vértice (ingreso en la Comunidad Europea) con respecto al primero (reivindicación sobre Gibraltar)-es evidente y se complica para los intereses españoles a causa de la guerra en el Atlántico Sur. Así, un editorial de El País (22 de junio) recoge la opinión dominante, de que el tema de Gibraltar encontrará más fácil solución en el marco de la Comunidad. Esta idea y el momentum negativo que ha generado la guerra -exacerbación del nacionalismo británico en relación con sus colonias- convierten el ingreso en la Comunidad Europea en objetivo prioritario.

De esta manera, el primer vértice del triángulo diplomático español (la reivindicación de Gibraltar) se ve subordinado al segundo vértice (el ingreso en la Comunidad Europea). Vértice que per se tiene, en palabras de Fernando Morán (1980: 289), un valor metapolítico. En éfecto, más allá de los intereses económicos, el ingreso de España en las Comunidades constituye un factor psicológico decisivo para la sociedad de la España democrática. Si tenemos en cuenta que, como si se tratara de una moneda, la transición a la democracia tiene su otra cara (la dimensión exterior), formada por el ingreso de España en la Comunidad. Así democratización y europeización van juntas. El complejo de inferioridad de los españoles -ligado a la imagen de 
una España atrasada, "bárbara", dictatorial y aislada de Europa-sólo puede curarse con su participación en el "club europeo". Así, la nueva identidad del español de 1982, el elemento que lo diferencia del pasado franquista, es su europeísmo. ${ }^{14}$

Ahora bien, la ruptura con el pasado no siempre es fácil, y menos en política exterior (caracterizada, en general, por su continuismo). Ahí es donde aparece el tercer vértice de la diplomacia española en 1982: las relaciones privilegiadas con América Latina.

Aquí nos encontramos con un tema de continuidad en la dimensión internacional de España. Si en el siglo XIX, los hispanistas habían defendido la existencia de una raza transatlántica, basada en la cultura y en el espíritu común; la España de Franco consigue a partir de la idea de la Hispanidad (de cariz católico y antidemocrático), la creación de una red de relaciones en América Latina -sustitutiva de sus relaciones con Europa (Morán, 1980: 18)-que aborda con gran pragmatismo a lo largo de los años (Pollack, 1987: 82; Wilson y Perfit, 1986: 171).

Influencia y "misión"15 (power y purpose) explican la política de España con América Latina, tanto durante el franquismo como en el momento que estalla la guerra de las Malvinas. En primer lugar, al igual que las otras ex-metrópolis, España utiliza las relaciones con sus antiguas colonias como un mecanismo para aumentar su influencia a nivel internacional (Mujal, 1986: 378). En segundo lugar, la "misión" española en América Latina es una constante que, bajo ideologías diferentes, encontramos en la España de Franco y en la España del 82. Así, los lazos en común entre las dos orillas del Atlántico se centran: según el conservador tradicionalista en la religión católica, según el demócrata en la lengua común y en la democratización, y según el izquierdista tercermundista en la resistencia contra el hegemonismo de los Estados Unidos. Los espacios entre unos y otros no son claros y, en buena medida, el latinoamericanismo de Falange comparte ideas con el tercermundismo de los izquierdis-

\footnotetext{
${ }^{14}$ Este tipo de proceso lo hemos visto también en la Europa Central y Oriental a partir de 1989, con la desaparición del bloque soviético y de los regímenes comunistas. Véase Waever, Ole, "Re-allocation of identities in Europe: The new configuration among EC, nation and region", Paper for the Anoual Mecting of the American Political Science Association, Chicago, septiembre 1992.

${ }^{15}$ Influencia y misión corresponde a los términos power y purpose. Ambos términos centran el análisis de la política exterior llevado a cabo por la escuela realista, destacando el caso de Hans J. Morgenthau. Sobre su uso en dicha teoría y traducción al español véase E. Barbé, "Estudio preliminar, traducción y notas", en: H. J. Morgenthau, Escritos sobre polftica internacional, (Madrid: Tecnos, 1990).
} 
tas, siendo en todos ellos la voluntad de diferencia con respecto al mundo anglosajón un factor central (Wiarda: 446). Adolfo Suárez es el más claro ejemplo de "hibridismo" ideológico en defensa de las relaciones especiales entre españoles y latinoamericanos. Lo que algunos autores han calificado de progresismo-populismo (Menéndez del Valle, 1989: 737).

Los constituyentes de la España democrática recogieron el carácter especial de dichas relaciones, cuando en el capítulo primero de la Constitución (1978) se habla de españoles, de iberoamericanos y de extranjeros. La doble nacionalidad, contemplada en la Constitución (art. 11.3), responde a una evidencia: el elevado número de latinoamericanos que disfrutan también de la nacionalidad española. Según fuentes periodísticas, en 1982, se cifraba su número en tres millones. En las mismas fechas, y a causa de los regímenes dictatoriales del Cono Sur, vivían en España aproximadamente 50.000 argentinos, 15.000 chilenos y 10.000 uruguayos en calidad de exiliados políticos. Estos factores sirven para reafirmar la continuidad en la España democrática de una realidad en términos humanos.

De hecho, durante las negociaciones de adhesión a la Comunidad Europea, España presentará sus relaciones privilegiadas con América Latina como una baza positiva, un elemento de prestigio. Es ilustrativo, en este sentido, el discurso de Pérez Llorca sobre el papel de España como puente entre Europa y América Latina. Ahora bien, la guerra de las Malvinas coloca, como hemos visto, al Gobierno español en una posición difícil que demuestra su incapacidad para actuar como puente (Arenal, 1989: 45). La realidad es que la doble dimensión de sus intereses -Europa y América Latinaparaliza la actuación del Gobierno. De tal manera que lo que antes era positivo (más prestigio), ahora es negativo (parálisis). De ahí la necesidad de optar, que hace imposible mantener la relación equidistante entre el segundo vértice (Comunidad Europea) y el tercer vértice (América Latina). De hecho, la lección que podemos extraer de la guerra y de las posteriores relaciones entre España y los dos contendientes (por un lado Gran Bretaña, más la Comunidad Europea, y por otro lado, Argentina, más el conjunto de América Latina) se inscribe en este punto. Es decir, en confirmar el papel subordinado del tercer vértice (las relaciones españolas con América Latina) respecto del segundo vértice (las relaciones con la Comunidad Europea). 
En resumen, y antes de abordar el apartado de las lecciones a extraer de la guerra y sus repercusiones, se puede concluir que la agenda diplomática española no tenía la forma de un triángulo equilátero con tres vértices separados por lados idénticos, sino que nos encontramos frente a un triángulo isósceles en el que el vértice comunitario domina claramente sobre los otros dos (Gibraltar y América Latina) gracias a su ubicación (predominio sobre los otros dos, de los que le separa una mayor distancia).

\section{V.- Las lecciones del comportamiento español en la postguerra.}

Una vez acabada la guerra en el Atlántico Sur, las Malvinas no desaparecen de la agenda diplomática española. Al contrario, la llegada de la democracia a Argentina (diciembre de 1983) supone la reaparición del tema. Tanto en 1983 como en 1984, la intervención del Ministro Morán en la Asamblea General de Naciones Unidas dedica unos segundos a la descolonización de las Islas. Es, sin embargo, la declaración hispano-argentina firmada en junio de 1984, durante la primera visita del Presidente Alfonsín a Madrid, la que mejor destaca la posición de ambos países. En el punto 6 de la ruisma se lee: "España y Argentina, que son víctimas de una anacrónica situación colonial, apoyan sus respectivas reivindicaciones de soberanía sobre las Islas Malvinas y Gibraltar para restaurar la integridad de sus territorios nacionales por la vía pacífica, de conformidad con las resoluciones pertinentes de las Naciones Unidas".

La declaración, que dio lugar a una condena de Margaret Thatcher en la Cámara de los Comunes (el 14 de junio), es el producto único de un momento muy concreto en las relaciones hispano-argentinas. El Presidente González halla en la Argentina democratizada un partenaire ideal para iniciar su andadura internacional. Junto a los factores histórico/sentimentales que unen a España y Argentina, González y Alfonsín introducirán una imagen de democracia y de derechos humanos, favorable para ambos líderes y para sus respectivas proyecciones internacionales. De hecho, el primer Gobierno de Felipe González (1982-1985) lleva a cabo una política presidencialista en América Latina (primer escenario, antes 
que Europa, de proyección de su imagen internacional) que se traduce en frecuentes viajes por la región.

La lógica identificadora entre Gibraltar y las Islas desaparece de la agenda del Gobierno español a partir del momento en que España y Gran Bretaña alcanzan el acuerdo de Bruselas (diciembre de 1984). El propio Fernando Morán dirá que el acuerdo de Bruselas sobre Gibraltar "no sirve de precedente ni para Ceuta y Melilla, ni para las Malvinas" (1984: 11). Lo que no significa que España modifique su actitud respecto a la descolonización de las Malvinas, como muestra su voto favorable en la Asamblea General de Naciones hasta 1988 (último año en que el tema es sometido a votación) o la importancia que Juan Carlos I da al tema durante su primera visita a la Argentina democrática en 1985. Ahora bien, el Gobierno español separa a partir de 1985 el tema de las Islas del tema de Gibraltar (sometido al marco bilateral o al marco comunitario) y pasa de ser un tema explícito e importante en las relaciones hispano-argentinas a convertirse en un parti pris en el marco de Naciones Unidas.

A partir de 1986, las relaciones hispano-argentinas están sometidas a una coyuntura diferente, dado el ingreso de España en la Comunidad Europea. Es evidente -y el discurso del Gobierno español sobre "el papel de puente", durante la guerra del 82, lo había evidenciado- que el ingreso de España en la Comunidad Europea había de tener consecuencias sobre las relaciones con América Latina. En ese sentido, tanto la derecha ligada al franquismo (Fraga Iribarne, 1982: 47) como los ideólogos de la diplomacia socialista (Yáñez, 1986: 249) han abordado las relaciones Comunidad Económica Europea/España/América Latina en términos de' lobby. Es decir, definiendo el papel de España como representante o correa de transmisión de los intereses latinoamericanos entre los Doce. En ese terreno se inscribe, en octubre de 1986, la reacción española contraria a la decisión británica de crear una zona de exclusión de 150 millas alrededor de las Malvinas en base, según una nota de la Oficina de Información Diplomática, a la posición española sobre la soberanía de las Islas. Ahóra bien, la condena española no se traduce posteriormente en una acción en el marco de la política pesquera de los Doce. Así cuando Gran Bretaña comienza a expedir las.licencias de pesca en la zona, España no pone objeciones, dados sus intereses económicos. Hay que recordar aquí que en 1986 España era el país comunitario con mayores intereses pesqueros en la zona (a la par con Unión Soviética, Japón, Taiwán, Polonia y Corea del Sur). 
Este caso ilustra el cambio que se va a producir en las relaciones hispano-argentinas a partir del ingreso español en la Comunidad. Dos grandes rasgos caracterizan el cambio: 1.- la política de ideologías compartidas (democratización, reivindicación territorial) da paso a una política de intereses económicos, en muchos casos divergentes y 2.- la política presidencialista de González en América Latina es sustituida por una política burocrática (Arenal, 1989: 34), más apropiada al nuevo tipo de relaciones (centradas en la cooperación económica), mientras Felipe González dedica mayor interés personal a sus relaciones con los países de la Comunidad Europea.

1.- El período 1982-1987 en las relaciones hispano-argentinas es calificado por los analistas como un período rico en coincidencias políticas, pero pobre en "negocios" (Russell, 1991: 13). Ello responde a una constante en las relaciones hispano-argentinas (y por extensión, latinoamericanas): la asimetría entre lo político y lo económico $o$, lo que es lo mismo, España tiene más influencia que poder, basado en recursos tangibles, en América Latina.

En efecto, el peso económico de España es pequeño (en 1980 el mercado español significaba el $3 \%$ del total de los intercambios comerciales de América Latina y el 3\% de la deuda latinoamericana estaba contraída con bancos españoles). A la inversa, las cifras son algo diferentes (en los momentos de mayor intercambio comercial, a principios de los ochenta, América Latina significa el 10\% del comercio español y, a nivel crediticio, el papel de América Latina es importante ya que, por las mismas fechas, el 50\% de los créditos españoles estaban concedidos a América Latina y, a Argentina en concreto el 17\%) (Falcoff, 1986).

Los intereses divergentes de España y Argentina aumentan con el ingreso de España en la Comunidad Europea y el impacto negativo que la misma tiene sobre la economía argentina. Tan sólo a nivel de relaciones bilaterales, hay que apuntar que entre 1984 y 1987 desaparece el superávit comercial de Argentina con España (cifrado en 1984 en 200 millones de dólares) y que las inversiones se reducen a la mitad entre 1985 y 1986 (pasando del 2,4\% al 1,2\% del total de las inversiones españolas en el exterior). Lo que responde a una evidencia (previa ya al ingreso en la Comunidad): la orientación hacia Europa de la economía española (en 1991, un 65\% de las importaciones españolas venían de la Comunidad Europea/EFTA frente a un 4,5\% de América Latina y un 75\% de las exportaciones españolas iban a la Comunidad Europea/EFTA frente a un 3,5\% a América Latina). 
2.- El carácter más desideologizado y más pragmático de las relaciones hispano-argentinas se verá fortàlecido con la llegada al poder de Menem, quien por su parte adopta una actitud más realista que el Gobierno radical en materia internacional. Lo que lleva, en el caso de las Malvinas, al restablecimiento de relaciones diplomáticas entre Argentina y Gran Bretaña (1990), tras las negociaciones de Madrid, iniciadas en $1989^{16}$

\section{VI.- Conclusiones.}

La evolución del comportamiento español durante la década que sigue a la guerra de las Malvinas nos muestra un cambio sustancial en los mecanismos de elaboración de la política exterior española. No en vano la década de los años ochenta coincide con el período de definición (contenido e instrumentos) de la política exterior de la España democrática. De ahí que el análisis de la guerra, por una parte, y el seguimiento de las relaciones de España con Argentina (por extensión con América Latina) y con Gran Bretaña (por extensión con la Comunidad Europea) en el período que va de 1982 a 1992, precisen de enfoques teóricos diferentes. ${ }^{17}$

En primer lugar, la elaboración de la política exterior española durante la guerra de 1982 responde a las circunstancias de España en aquel momento: la indefinición del país en materia de política exterior junto a la inexistencia de un aparato burocrático asentado. $\mathrm{El}$ análisis del comportamiento español durante la guerra nos lleva a dos conclusiones de carácter general:

1.- El Gobierno de Calvo Sotelo actúa de manera ambigua a lo largo de la guerra. Por una parte, actúa siguiendo pautas de actor racional (las negociaciones con la Comunidad Económica Europea como preferencia clara) y, por otra parte, se ve afectado por las ideas (belief-systems) que, a través de una opinión pública claramente pro-argentina, inciden en los decisores (decision makers). Dicha ambigüedad, junto con la falta de cartas del Gobierno español (no

\footnotetext{
${ }^{16}$ A pesar de la elección de la capital de España como "espacio negociador", el Gobierno español se halla totalmente al margen de la iniciativa y del desarrollo de dichos encuentros.

${ }^{17}$ Sobre los elementos de foreign policy analysis, teorías y métodos, mencionados en estas conclusiones, véase J. Greenaway, S. Smith, y I. Street, Deciding factors in British politics - $A$ case-studies approach, (London: Routledge, 1992). Junto a las traducciones en español, se incluyen los términos originales de los autores.
} 
pertenencia a la Comunidad), invalidan por completo las propuestas españolas de jugar el papel de mediador entre Europa y América Latina a lo largo de la crisis.

2.- La guerra se convierte en un pretexto para los partidos de la oposición que, como en un modelo de política de adversarios (adyersarial politics), ${ }^{18}$ aprovechan la ocasión para rebatir la orientación dada por Calvo Sotelo a la política exterior. Al mismo tiempo, la oposición convierte la guerra de las Malvinas en un episodio más de la batalla política interna entre partidos e incluso en el seno del propio partido en el Gobierno (UCD), que va a acabar con la victoria por mayoría absoluta del PSOE en las elecciones de octubre de 1982.

En segundo lugar, el seguimiento de la política española en relación con el conflicto de las Malvinas desde la llegada de los socialistas al poder se enmarca en el conjunto de las relaciones de España, al mismo tiempo, con Argentina (América Latina) y con Gran Bretaña (Comunidad Europea). De lo que se pueden extraer cuatro conclusiones de carácter general:

a) El debate entre las fuerzas políticas españolas con ocasión de la guerra del Atlántico Sur, que se ajusta al modelo de política de adversarios (adversarial politics), supone que la llegada al poder de la oposición (PSOE) comportará un cambio de política en relación con las Malvinas y con Argentina. El cambio es de orientación, ya que la política latinoamericana de Felipe González va a estar marcada en un principio por elementos ideológicos (democratización) y no por elementos históricos tradicionales, como en etapas anteriores, Lo que convierte a Argentina (a partir de la caída de la Junta Militar) en uno de los partenaires principales del Gobierno español en América Latina. Dentro de esa relación privilegiada entre España y Argentina, el tema de las Malvinas juega un papel simbólico como reforzador del Gobierno de Alfonsín.

b) El Gobierno González, al igual que le ocurrió al Gobierno Calvo Sotelo, toma decisiones en materia exterior por el cálculo racional que lleva a España a considerar la Comunidad Europea como el espacio preferido en todos los sentidos (económico, político, marco para favorecer la solución del conflicto de Gibraltar, etc.), pero al mismo tiempo se ve influido por las ideas (belief-systems) de la sociedad española que hacen de América Latina "un mundo propio". De ahí la retórica de los socialistas españoles sobre el papel de

\footnotetext{
${ }^{18} \mathrm{El}$ modelo de adversarial politics se interesa por las relaciones entre oposición y Gobiemo, conducentes al cambio o discontinuidad en poítica exterior.
} 
España como puente entre América Latina y los países de la Comunidad Europea.

c) El Gobierno González va perfilando a lo largo de la década que sigue a la guerra de 1982 un modelo para articular las relaciones de España, al mismo tiempo, con la Comunidad Europea y con América Latina. Así, a partir del ingreso español en la Comunidad (1986) se da un doble proceso: la europeización de la política latinoamericana del Gobierno español y la articulación, gracias a la actividad diplomática española, de una región iberoamericana. En el primer caso, la europeización de la política española en América Latina ha supuesto que España tuviera que renunciar a políticas propias, como en Nicaragua a partir de 1983 (Grugel, 1987), pero al mismo tiempo ha convertido a España en el portavoz de los latinoamericanos en el marco de la Cooperación Política Europea (Barbé, 1990). En el ségundo caso, se ha fraguado la voluntad de potenciar un espacio regional iberoamericano, basado en una realidad político-social (no económica). Esta realidad política se basa en una identidad positiva (histórica, sentimental y cultural) y en una identidad negativa (diferenciarse del mundo anglosajón).

d) El objetivo español de potenciar la creación de una región iberoamericana ha supuesto una mayor complejidad y burocratización de la elaboración de la política exterior (foreign policy making). En especial, a partir de 1985, momento en que Felipe González orienta su agenda personal hacia las relaciones con Europa, en perjuicio de sus contactos personales en América Latina. Se puede decir que a partir de 1985 (Morán es sustituido por Fernández Ordóñez en el Ministerio de Asuntos Exteriores), la elaboración de la política de España hacia Latinoamérica se transforma, pasando de la dirección desde el Gobierno (cabinet government) a la normalización burocrática (bureaucratic dispersion), con la consiguiente distribución de funciones.

Esta distribución supone que el Rey asuma el contenido simbólico de la política española en América Latina. Así, el monarca se convierte en el portavoz del aparato ideológico-cultural, la Comunidad Tberoamericana de Naciones (Mesa, 1989; Arenal, 1989). Al mismo tiempo se crea una estructura institucional que aúna cooperación internacional y América Latina: una Secretaría de Estado para la Cooperación Internacional e Iberoamérica, la Agencia Española de Cooperación Internacional y el Plan Anual de Cooperación Internacional. La existencia en paralelo de varios órganos encargados 
durante los últimos años de las relaciones con América Latina y cada uno de ellos en manos de un grupo diferente (asesores de Felipe González, diplomáticos del Ministerio, miembros del Partido Socialista encargados de los mecanismos de cooperación) ha complicado el proceso organizativo (organisational process), de tal manera que los acontecimientos de 1992 (Exposición de Sevilla, Celebración del Quinto Centenario, II Conferencia Iberoamericana de Madrid) han sido el marco de luchas de poder entre los diversos grupos de interés encargados de la elaboración de la política española con América Latina.

e) Finalmente, una década de experiencia, desde la guerra de las Malvinas, muestra que, a pesar de la preferencia indiscutible (alinearse en todos los sentidos con el mundo comunitario), no hay que infravalorar el peso de los valores (espacio cultural y sentimental iberoamericano) en momentos de crisis, tal y como volvió a reflejar la intervención de Estados Unidos en Panamá (1989). En aquella ocasión, España rompió con la disciplina de voto de los Doce en la Asamblea General de Naciones Unidas para condenar, junto a los países iberoamericanos, la actuación de Estados Unidos. En suma, una década después de la guerra de las Malvinas, España -gran defensor de la diplomacia europea-es todavía un disidente potencial, cuando se trata de conflictos armados que afectan a países de América Latina. Lo cual no constituye ninguna novedad en el marco de los Doce, si tenemos en cuenta que las potencias ex-coloniales de la Comunidad siempre han reforzado sus vínculos tradicionales, allende del continente, a pesar de que ello afectara negativamente a la solidaridad europea. Esta actitud se ha visto agravada, al trasladarse las divergencias al interior del continente europeo (la creación de nuevos Estados a partir de la desmembración de Yugoslavia).

En suma, España ha normalizado a lo largo de la última década su política exterior en las direcciones abordadas en estas páginas: por un lado, ha burocratizado sus relaciones con América Latina y, por otro lado, ha defendido su prestigio internacional (influencia simbólica en América Latina) y, con él, sus intereses nacionales a costa de la solidaridad europea. Solidaridad, todo hay que decirlo, que padece una fuerte crisis desde el fin de la guerra. Por supuesto, la Guerra Fría y no la de las Malvinas. 


\section{BIBLIOGRAFIA}

Arenal, C. del (1989), España e Iberoamérica, de la Hispanidad a la Comunidad Iberoamericana de Naciones, (Madrid: Cedeal).

Armero, J. M. (1989), Política exterior de España en democracia, (Madrid: Espasa Calpe).

Barbé, E. (1990), "El año español de la Cooperación Política Europea", Anuario Internacional CIDOB 1989, Barcelona, CIDOB, pp. 109-120.

Barbé, E. (1990a), "La transición española: Cambio y continuidad en la política exterior y de seguridad", Papers, No33, pp. 103-120.

Bendahmane, D. B. y Mc Donald, J. W. (1986), "Mediation attempts in the Falklands/Malvinas crisis", Perspectives on negotiation. Four case studies and interpretations, Washington, Foreigi Service Institute, pp. 51-98.

Calvo Sotelo, L. (1990), Memoria viva de la transición, (Barcelona: Plaza Janés).

Castiella, F. M. (1941), Reivindicaciones de España, (Madrid: Instituto de Estudios Políticos).

Cortada, J. W. (ed.) (1980), Spain in the twentieth-century world. Essays on Spanish Diplomacy 1898-1978, (London: Aldwych Press).

Edwards, G. (1984), "Europe and the Falkland Islands Crisis", Joumal of Common Market Studies, vol. XXII, N4, pp. 295-313.

FernándezSánchez, P. A. (1984), "La crisis de las Malvinas ante las Naciones Unidas", Revista de Estudios Internacionales, vol. 5, N4, pp. 923-953.

Fraga Iribarne, M. (1982), España, entre dos modelos de sociedad, (Barcelona: Planeta).

Groom, J. R. (1991), "L'évolution de la question de Gibraltar", en: M. Rousset (ed.), La Méditerranée Occidentale, espace de coopération, (Grenoble: CEDSI), pp. 58-73.

Grugel, J. (1987), "Spain's socialist government and Central American dilemmas", Intemational Affairs, vol. 63, №4, pp. 603-615.

Marquina Barrio, A. (1986), España en la política de seguridad occidental, (Madrid: Ejército).

Martín Villa, R. (1984), Al servicio del Estado, (Barcelona: Planeta).

Mesa, R. (1985), "El proceso de toma de decisiones en política exterior", Documentación administrativa, N2205, pp. 143-163.

Mesa, R. (1988), Democracia y política exterior en España, (Madrid: Eude$\mathrm{ma})$.

Mesa, R. (1989), La idea de Comunidad Iberoamericana: entre la historia y la utopía, (Madrid: Cedeal).

Morán, F. (1980), Una politica exterior para España, (Barcelona: Planeta).

Morán, F. (1990), España en su sitio, (Barcelona: Plaza y Janés). 
E. Barbé / Entre Europa y América Latina: la diplomacia ...

Morodo, R. (1984), La transición política, (Madrid: Tecnos).

Morris D. S. and Haigh, R. H. (1992), Britain, Spain and Gibraltar 1945-90, (London: Routledge).

Mujal León, E. (1986), "Spain and Latin América: The Quest for Partnership", en H. J. Wiarda (op. cit.), pp. 375-407.

Oreja, M. (1976), "Discurso de Marcelino Oreja en la XXI Asamblea General de Naciones Unidas (27-9-1976) ${ }^{n}$, en: Tezanos, J. F. et al. (eds.), La transición democrática española, (Madrid: Sistema, 1989).

Pérez Llorca, P. (1982), "Intervención del Ministro de Asuntos Exteriores ante la Comision de Asuntos Exteriores del Congreso sobre el problema de las Malvinas", Actividades, textos y documentos de la política exterior española 1982, Madrid, Oficina de Información Diplomática, pp. 124-129.

Pollack, B. (1987), The paradox of Spanish Foreign Policy. Spain's International Relations from Franco to Democracy, (Londres: Pinter Publishers).

Preston, P. y Smith, D. (1985), España ante la CEE y la OTAN, (Barceliona: Grijalbo).

Remiro Brotons, A. (1984), La acción exterior del Estado, (Madrid: Tecnos).

Rupérez, J. (1986), España en la oTAN, (Barcelona: Plaza y Janés).

Russell, R. (1991), Las relaciones de Argentina con Europa Occidental, (Madrid: Irela).

Saba, K. (1986), "The Spanish Foreign Policy Decision-Making Process", The International Spectator, vol. XXI, No4, pp. 24-33.

Stavridis, S. (1992), "Blood is thicker than water: The reaction in Spain to the Falklands war of 1982", (texto no publicado).

Wiarda, H. J. (ed.) (1986), The Iberian-Latin American Connection. Implications for U.S. Foreign Policy, (Boulder: Westview Press).

Wiarda, H. J. (1986), "The Relations of Iberia and Latin-América and Some Implications for the United States", en: H. J. Wiarda, op. cit., pp. 427-446.

Wilson, L. C. and Perfit J. T. (1986), "Spain and Latin América: Diplomatic and Military Ties", en: H. J. Wiarda, op. cit., pp. 170-208.

Yáñez Barnuevo, J. A. (1986), "Relaciones entre Europa e Iberoamérica en el marco de las relaciones Norte-Sur y Este-Oeste", en: Encuentros en la Democracia: Europa-Iberoamérica, (Madrid: ICI). 